\title{
Operant Conditioning of Rat Soleus H-Reflex Oppositely Affects Another H-Reflex and Changes Locomotor Kinematics
}

\author{
Yi Chen, Lu Chen, Yu Wang, Jonathan R. Wolpaw, and Xiang Yang Chen \\ Laboratory of Neural Injury and Repair, Wadsworth Center, New York State Department of Health and State University of New York at Albany, Albany, \\ New York 12201-0509
}

H-reflex conditioning is a model for studying the plasticity associated with a new motor skill. We are exploring its effects on other reflexes and on locomotion. Rats were implanted with EMG electrodes in both solei $\left(\mathrm{SOL}_{\mathrm{R}}\right.$ and $\left.\mathrm{SOL}_{\mathrm{L}}\right)$ and right quadriceps $\left(\mathrm{QD}_{\mathrm{R}}\right)$, and stimulating cuffs on both posterior tibial (PT) nerves and right posterior femoral nerve. When $S O L_{R} E M G$ remained in a defined range, $\mathrm{PT}_{R}$ stimulation just above M-response threshold elicited the $\mathrm{SOL}_{R} \mathrm{H}$-reflex. Analogous procedures elicited the $\mathrm{QD}_{\mathrm{R}}$ and $\mathrm{SO} \mathrm{L}_{\mathrm{L}} \mathrm{H}$-reflexes. After a control period, each rat was exposed for $50 \mathrm{~d}$ to a protocol that rewarded $S O \mathrm{~L}_{\mathrm{R}} \mathrm{H}$-reflexes that were above (HRup rats) or below (HRdown rats) a criterion. HRup conditioning increased the $\mathrm{SOL}_{\mathrm{R}} \mathrm{H}$-reflex to $214 \pm 37 \%$ (mean $\pm \mathrm{SEM}$ ) of control $(p=0.02)$ and decreased the $\mathrm{QD}_{\mathrm{R}} \mathrm{H}$-reflex to $71 \pm 26 \%(p=0.06)$. HRdown conditioning decreased the $\mathrm{SOL}_{\mathrm{R}} \mathrm{H}$-reflex to $69 \pm 2 \%(p<0.001)$ and increased the $\mathrm{QD}_{\mathrm{R}}$ $\mathrm{H}$-reflex to $121 \pm 7 \%(p=0.02)$. These changes remained during locomotion. The $\mathrm{SOL}_{\mathrm{L}} \mathrm{H}$-reflex did not change. During the stance phase of locomotion, ankle plantarflexion increased in HRup rats and decreased in HRdown rats, hip extension did the opposite, and hip height did not change. The plasticity that changes the $\mathrm{QD}_{\mathrm{R}} \mathrm{H}$-reflex and locomotor kinematics may be inevitable (i.e., reactive) due to the ubiquity of activity-dependent CNS plasticity, and/or necessary (i.e., compensatory) to preserve other behaviors (e.g., locomotion) that would otherwise be disturbed by the change in the $\mathrm{SOL}_{\mathrm{R}} \mathrm{H}$-reflex pathway. The changes in joint angles, coupled with the preservation of hip height, suggest that compensatory plasticity did occur.

\section{Introduction}

The spinal stretch reflex (SSR) (i.e., the tendon jerk) and its electrical analog, the H-reflex, are the simplest motor behaviors. They are produced primarily by a two-neuron, monosynaptic pathway comprised of the primary afferent fiber, its synapse on the motoneuron, and the motoneuron itself (Wolpaw et al., 1983; Wolpaw, 1987). Because it is affected by descending activity from the brain, this pathway can be operantly conditioned. In response to a conditioning protocol, monkeys, humans, rats, and mice can gradually increase (i.e., up-conditioning) or decrease (i.e., downconditioning) the SSR or the H-reflex (for review, see Wolpaw, 2010). The larger or smaller reflex that results is a simple motor skill [i.e., "an adaptive behavior acquired through practice" (Chen et al., 2005)]. H-reflex conditioning is accompanied by

Received March 25, 2011; revised June 10, 2011; accepted June 21, 2011.

Author contributions: Y.C., L.C., Y.W., J.R.W., and X.Y.C. designed research; Y.C., L.C., Y.W., J.R.W., and X.Y.C. performed research; Y.C., L.C., Y.W., J.R.W., and X.Y.C. analyzed data; Y.C., L.C., Y.W., J.R.W., and X.Y.C. wrote the paper.

This work was supported by NIH Grants NS061823 (J.R.W., X.Y.C.), HD36020 (X.Y.C.), and NS22189 (J.R.W.), and the New York State Spinal Cord Injury Research Trust Fund (X.Y.C.). We thank Rongliang Liu for excellent technical assistance, and Drs. Jonathan S. Carp, Dennis J. McFarland, and Elizabeth Winter Wolpaw for valuable comments on this manuscript.

Correspondence should be addressed to either Yi Chen or Xiang Yang Chen, Laboratory of Neural Injury and Repair, Wadsworth Center, New York State Department of Health, P.0. Box 509, Albany, NY 12201-0509. E-mail: chenyi@wadsworth.org or chenx@wadsworth.org.

DOI:10.1523/JNEUROSCI.1526-11.2011

Copyright $\odot 2011$ the authors $\quad 0270-6474 / 11 / 3111370-06 \$ 15.00 / 0$ neuronal and synaptic plasticity at multiple sites in the spinal cord and brain (for review, see Wolpaw and Chen, 2009).

$\mathrm{H}$-reflex conditioning is a powerful model for exploring the mechanisms and principles of skill acquisition and maintenance (Wolpaw, 2010). The spinal cord is the final common pathway for all motor behavior, and spinal cord plasticity has a part in the acquisition and maintenance of many motor skills. Furthermore, by virtue of their simplicity, accessibility, separation from the brain, and closeness to behavior, the spinal cord in general and the H-reflex in particular are uniquely suited for studying how activity-dependent plasticity (particularly gradual plasticity) explains behavior, and for formulating concepts and identifying principles that may apply to learning throughout the CNS.

Because the spinal cord is the final common pathway for motor output, the spinal cord plasticity associated with H-reflex conditioning affects other behaviors. For example, in normal rats, right soleus H-reflex up- and down-conditioning produce corresponding changes in the right soleus burst during locomotion (Chen et al., 2005). Nevertheless, despite this change, the right/left symmetry of the step cycle is preserved. This suggests that changes in other reflex pathways compensate for the locomotor effects of the change in the soleus H-reflex pathway. This hypothesis is supported by other evidence that the functional effects of $\mathrm{H}$-reflex conditioning extend beyond the conditioned reflex, and even to the contralateral side of the spinal cord (Wolpaw and Lee, 1989).

The present study is the first effort to determine whether soleus H-reflex conditioning affects the H-reflex of a hindlimb 
muscle group [i.e., quadriceps (QD)] that operates about different joints (i.e., knee and hip rather than ankle), and whether it affects joint angles during locomotion. The data show that soleus $\mathrm{H}$-reflex conditioning has a markedly different effect on the QD $\mathrm{H}$-reflex and produces distinctive multijoint kinematic changes during locomotion. They indicate that the acquisition of an ostensibly simple new skill has an impact that extends beyond the new skill to affect a crucial older skill, and they raise important new questions.

\section{Materials and Methods}

Twenty-six male Sprague Dawley rats [ $439 \pm 44 \mathrm{~g}$ (mean $\pm \mathrm{SD}$ ) initially] were studied. All procedures satisfied the Guide for the Care and Use of Laboratory Animals, and had been approved by the Wadsworth Center Institutional Animal Care and Use Committee. The methods have been fully described previously (Chen and Wolpaw, 1995, 2002; Chen et al., 2005; X. Y. Chen et al., 2006; Y. Chen et al., 2006) and are summarized here.

Electrode implantation. Under general anesthesia [ketamine $\mathrm{HCl}(80$ $\mathrm{mg} / \mathrm{kg}$ ) and xylazine $(10 \mathrm{mg} / \mathrm{kg}$ ) (both i.p.) or sodium pentobarbital (60 $\mathrm{mg} / \mathrm{kg}$, i.p.), supplemented as needed] and aseptic conditions, each rat was implanted with chronic stimulating and recording electrodes in the right and left legs. To elicit $\mathrm{H}$-reflexes in the right and left solei $\left(\mathrm{SOL}_{\mathrm{R}}\right.$ and $\left.\mathrm{SOL}_{\mathrm{L}}\right)$, cuffs were placed on the right and left posterior tibial $\left(\mathrm{PT}_{\mathrm{R}}\right.$ and $\mathrm{PT}_{\mathrm{L}}$ ) nerves just above the triceps surae branches. To elicit the H-reflex of the right quadriceps muscle group $\left(\mathrm{QD}_{\mathrm{R}}\right)$, a similar cuff was placed on the right posterior division of the femoral $\left(\mathrm{PF}_{\mathrm{R}}\right)$ nerve. To record $\mathrm{SOL}_{\mathrm{R}}$, $\mathrm{SOL}_{\mathrm{L}}$, and $\mathrm{QD}_{\mathrm{R}} \mathrm{EMG}$, a pair of stainless steel fine-wire electrodes was implanted in each. The soleus acts about the ankle joint to plantarflex the foot. The QD group comprises four muscles: vastus lateralis, medialis, and intermedialis, and rectus femoris. All four act about the knee to dorsiflex (i.e., extend) the calf, and the rectus femoris also acts about the hip to flex the thigh (i.e., to move it forward and up). The two $\mathrm{QD}_{\mathrm{R}} \mathrm{EMG}$ electrodes were placed laterally (targeting vastus lateralis) and medially (targeting vastus medialis), respectively, so that their data would represent the entire QD muscle group. The Teflon-coated wires from all the electrodes passed subcutaneously to a connector on the skull.

After surgery, the rat was placed under a heating lamp and given an analgesic (Demerol, $0.2 \mathrm{mg}$, intramuscular). Once awake, it received a second dose of analgesic and was returned to its cage and allowed to eat and drink ad libitum.

Experimental design and $S O L_{R} H$-reflex conditioning. Data collection began at least $20 \mathrm{~d}$ after surgery and continued $24 \mathrm{~h} / \mathrm{d}, 7 \mathrm{~d} /$ week for at least $70 \mathrm{~d}$. During this period, the rat lived in a standard rat cage with a 40 $\mathrm{cm}$ flexible cable attached to the skull connector. The cable, which allowed the animal to move freely about the cage, connected to a commutator above the cage that connected to EMG amplifiers (gain 1000, bandwidth $100-1000 \mathrm{~Hz}$ ) and the nerve-cuff stimulation units. The rat had ad libitum access to water and food, except that during H-reflex conditioning it received food mainly by performing the task described below. Animal well-being was carefully checked several times each day, and body weight was measured weekly. Laboratory lights were dimmed from 9:00 P.M. to 6:00 A.M. daily.

Stimulus delivery and data collection were controlled by a computer, which sampled ( $5 \mathrm{kHz}) \mathrm{SOL}_{\mathrm{R}}, \mathrm{SOL}_{\mathrm{L}}$, and $\mathrm{QD}_{\mathrm{R}} \mathrm{EMG}$ continuously for the entire period of study. $\mathrm{SOL}_{R}$ and $\mathrm{SOL}_{\mathrm{L}} \mathrm{H}$-reflexes were elicited simultaneously as follows. Whenever the absolute value (i.e., the full-wave rectified value) of background (i.e., ongoing) EMG in each muscle remained within a predefined range for a randomly varying $2.3-2.7 \mathrm{~s}$ period, the computer initiated a trial. In each trial, the computer stored the most recent $50 \mathrm{~ms}$ of EMG from all three muscles (i.e., the background EMG interval), delivered simultaneous monophasic stimulus pulses through the cuffs on the $\mathrm{PT}_{\mathrm{R}}$ and $\mathrm{PT}_{\mathrm{L}}$ nerves, and stored the EMG from all muscles for another $100 \mathrm{~ms}$. A comparable procedure elicited the $\mathrm{QD}_{\mathrm{R}}$ $\mathrm{H}$-reflex by stimulating the $\mathrm{PF}_{\mathrm{R}}$ nerve whenever ongoing $\mathrm{QD}_{\mathrm{R}} \mathrm{EMG}$ remained within a predefined range. Because SOL and QD H-reflex trials occurred only when the muscles satisfied their background EMG re- quirements, SOL and QD H-reflex trials seldom occurred in close proximity to each other.

Stimulus pulse amplitude and duration were initially set to produce a maximum $\mathrm{H}$-reflex (and an $\mathrm{M}$ response that was typically just above threshold) in the muscle served by the stimulated nerve. Pulse duration remained fixed (typically $0.5 \mathrm{~ms}$ ). After each trial, pulse amplitude was adjusted automatically to maintain the $\mathrm{M}$ response [i.e., average EMG amplitude in the $\mathrm{M}$ response interval (typically $2.0-5.0 \mathrm{~ms}$ in $\mathrm{SOL}_{\mathrm{R}}$ and $\mathrm{SOL}_{\mathrm{L}}$ and 1.5-4.5 $\mathrm{ms}$ in $\mathrm{QD}_{\mathrm{R}}$ )] unchanged throughout data collection. [This ensured that the effective strength of the stimulus was stable throughout (Wolpaw, 1987; Chen and Wolpaw, 1995).] H-reflex size was defined as average EMG amplitude in the H-reflex interval (typically 6-10 $\mathrm{ms}$ in $\mathrm{SOL}_{\mathrm{R}}$ and $\mathrm{SOL}_{\mathrm{L}}$ and 4.5-8.5 $\mathrm{ms}$ in $\mathrm{QD}_{\mathrm{R}}$ ) minus the muscle's average background EMG amplitude.

Under the control mode, the computer simply digitized and stored the EMG from each muscle for $100 \mathrm{~ms}$ following the stimulus. Under the $\mathrm{SOL}_{\mathrm{R}}$ up-conditioning (HRup) or down-conditioning (HRdown) mode, it also gave a food pellet reward $200 \mathrm{~ms}$ after the $\mathrm{PT}_{\mathrm{R}}$ nerve stimulation if the average amplitude of $\mathrm{SOL}_{\mathrm{R}}$ EMG in the $\mathrm{H}$-reflex interval was above (HRup) or below (HRdown) a criterion. The criterion was set and adjusted daily as needed, so that the rat received an adequate amount of food ( $\sim 1000$ pellets/d for a $500 \mathrm{~g}$ rat).

Each rat was first studied under the control mode for $\sim 20 \mathrm{~d}$. It was then exposed to $\mathrm{SOL}_{\mathrm{R}}$ up-conditioning (HRup rats) or downconditioning (HRdown rats) for $50 \mathrm{~d}$. The last 10 control-mode days and the last 10 HRup or HRdown days (i.e., days 41-50 of conditioning) provided the data used to assess the impact of $\mathrm{SOL}_{R} \mathrm{H}$-reflex conditioning on $\mathrm{SOL}_{\mathrm{R}}, \mathrm{QD}_{\mathrm{R}}$, and $\mathrm{SOL}_{\mathrm{L}} \mathrm{H}$-reflexes.

$H$-reflex and kinematic measurements during treadmill locomotion. $\mathrm{SOL}_{\mathrm{R}}$ and $\mathrm{QD}_{\mathrm{R}}$ locomotor H-reflexes and right hindlimb kinematics were studied in two treadmill sessions, one during the control-mode days and one near the end of $\mathrm{SOL}_{\mathrm{R}}$ HRup or HRdown conditioning. Treadmill speed was the same in both sessions (typically $10-12 \mathrm{~m} / \mathrm{min}$ ), and EMG was continuously recorded $(0.1-1.0 \mathrm{kHz}$ bandpass), digitized (4.0 $\mathrm{kHz}$ ), and stored. In each session, reflex data were collected for two 4-5 min periods. In one, the $\mathrm{PT}_{\mathrm{R}}$ nerve was stimulated to elicit the $\mathrm{SOL}_{\mathrm{R}}$ H-reflex, and in the other the $\mathrm{PF}_{\mathrm{R}}$ nerve was stimulated to elicit the $\mathrm{QD}_{\mathrm{R}}$ $\mathrm{H}$-reflex. The nerve was stimulated when its muscle's EMG had remained in a specified high range for $200 \mathrm{~ms}$. Thus the stimulus typically occurred in the later part of the right stance phase of the step cycle ( $\sim 100 \mathrm{~ms}$ past the middle of the muscle's locomotor burst). Stimulus amplitude was kept just above M-response threshold as described above. (H-reflex elicitation during stance meant that the reflex was measured at a time when its pathway is likely to affect locomotion.) In addition, the rat was videotaped (60 frames/s) from the right side during 4-5 min of treadmill walking without nerve stimulation.

Those trials for which $\mathrm{SOL}_{\mathrm{R}}$ or $\mathrm{QD}_{\mathrm{R}}$ EMG amplitude for the $20 \mathrm{~ms}$ immediately before the stimulus and $\mathrm{M}$-response size satisfied specified criteria were averaged by triggering on the stimuli. Thus, the average prestimulus EMG amplitudes and M-response sizes were the same for the two treadmill sessions.

In video analysis, we identified the right stance-phase images and, using marks on the ankle, knee, and hip, calculated for the entire stance phase the average anterior hip angle (i.e., hip extension: the angle of the thigh to a horizontal line projecting forward from the hip), posterior knee angle, and anterior ankle angle (i.e., ankle plantarflexion), and the average height of the hip above the treadmill surface.

Statistical analysis. The data fell into three categories. The first category consisted of $\mathrm{SOL}_{\mathrm{R}}, \mathrm{QD}_{\mathrm{R}}$, and $\mathrm{SOL}_{\mathrm{L}} \mathrm{H}$-reflexes elicited under the conditioning protocol, that is, throughout the day as the rat moved freely about its home cage. These are henceforth called "conditioning H-reflexes." The second category consisted of $\mathrm{SOL}_{\mathrm{R}}$ and $\mathrm{QD}_{\mathrm{R}} \mathrm{H}$-reflexes during the right stance phase of locomotion. These are called "locomotor H-reflexes." The third category consisted of average right stance-phase ankle, knee, and hip angles and average hip heights.

For each conditioning reflex, a paired $t$ test compared the average value for the last $10 \mathrm{~d}$ of $\mathrm{SOL}_{\mathrm{R}}$ HRup or HRdown conditioning to that for the last 10 control-mode days. For each locomotor reflex and joint angle and 


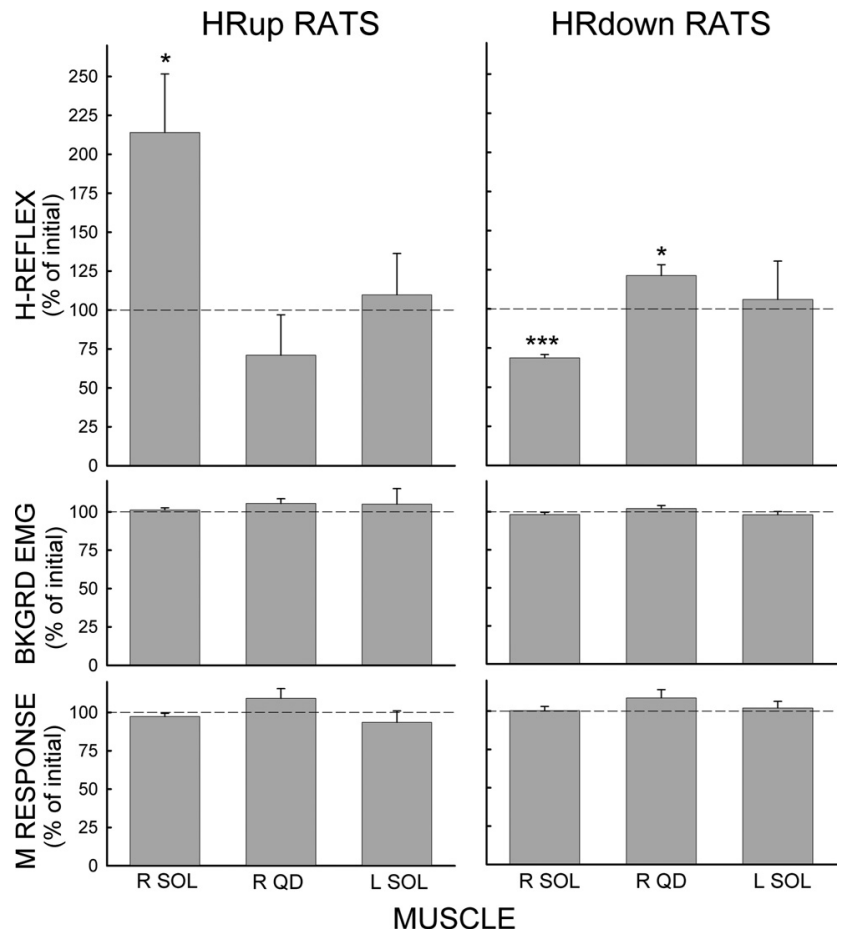

Figure 1. Effects of $S O L_{R}$ HRup and HRdown conditioning. Average final conditioning $H$-reflex, background EMG, and $M$ response (in percentage of their initial values) of $S O L_{R^{\prime}}, Q D_{R^{\prime}}$ and $\mathrm{SO}_{1}$ for the successful $\mathrm{HRup}$ and $\mathrm{HRdown}$ rats. $p$ values for difference from initial value by paired $t$ test are shown. ${ }^{*} p<0.05 ;{ }^{* * *} p<0.001$.

for hip height, a paired $t$ test compared the value for the second treadmill session to that for the first treadmill session.

Animal perfusion and anatomical study. At the end of study, each rat received an overdose of sodium pentobarbital (i.p.) and was perfused through the heart. The EMG electrodes, nerve cuffs, and PT and PF nerves were examined and the SOL muscles of both sides were removed and weighed.

\section{Results}

Animals remained healthy and active throughout study. Body weight increased from $439 \pm 44 \mathrm{~g}$ (mean $\pm \mathrm{SD}$ ) at implantation to $568 \pm 54 \mathrm{~g}$ at perfusion. In all rats, postmortem examination found that the cuffs were in place and covered by connective tissue, and that the nerves were well preserved. $\mathrm{SOL}_{\mathrm{R}}$ and $\mathrm{SOL}_{\mathrm{L}}$ weights did not differ significantly, nor did they differ from those of 113 normal rats previously studied.

\section{Effects of $\mathrm{SOL}_{\mathrm{R}} \mathrm{H}$-reflex conditioning on $\mathrm{SOL}_{\mathrm{R}}, \mathrm{QD}_{\mathrm{R}}$, and $\mathrm{SOL}_{\mathrm{L}}$ conditioning H-reflexes}

To determine the final effect on each muscle's conditioning H-reflex size of $\mathrm{SOL}_{\mathrm{R}}$ HRup or HRdown conditioning, the muscle's average H-reflex size for the final $10 \mathrm{~d}$ of conditioning was calculated as percentage of its initial $\mathrm{H}$-reflex size (i.e., average of final 10 control-mode days). As in previous studies, successful $\mathrm{SOL}_{\mathrm{R}} \mathrm{H}$-reflex conditioning was defined as a change $\geq 20 \%$ in the correct direction. By this criterion, $\mathrm{SOL}_{\mathrm{R}} \mathrm{H}$-reflex conditioning was successful in 9 HRup and 8 HRdown rats. In the remaining rats ( 4 HRup and $5 \mathrm{HRdown}$ ), the final $\mathrm{SOL}_{\mathrm{R}} \mathrm{H}$-reflex was within $20 \%$ of its initial value.

Figure 1 shows the average final values $( \pm \mathrm{SEM})$ of $\mathrm{SOL}_{\mathrm{R}}$, $\mathrm{QD}_{\mathrm{R}}$, and $\mathrm{SOL}_{\mathrm{L}} \mathrm{H}$-reflexes, $\mathrm{M}$ responses, and background $\mathrm{EMG}$ in the successful HRup (left) and HRdown (right) rats in percentage of their initial values. In accord with the goal of the condi-
HRup RAT

HRdown RAT

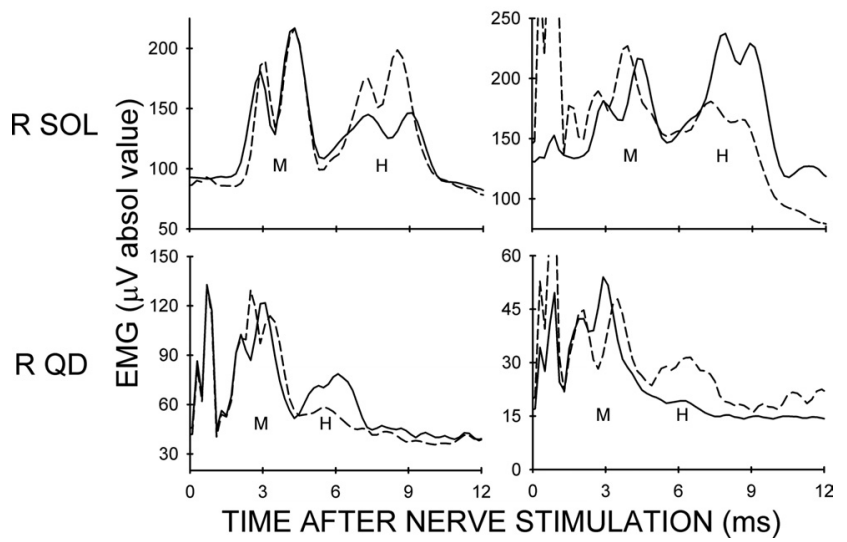

Figure 2. Effects of $S O L_{R} H R u p$ and HRdown conditioning on the conditioning H-reflexes of representative rats. Average poststimulus $S O L_{R}$ and $Q D_{R} E M G$ for a control day (solid) and a day near the end of conditioning (dashed) for an HRup rat and an HRdown rat. After HRup conditioning the $\mathrm{SO}_{R}$ $\mathrm{H}$-reflexis larger and the $\mathrm{QD} \mathrm{D}_{R} \mathrm{H}$-reflexis smaller, while after HRdown conditioning the $\mathrm{SO}_{\mathrm{R}} \mathrm{H}$-reflex is smaller and the $Q D_{R} H$-reflex is larger. Background EMG (EMG at time 0 ) and $M$-responses do not change. Peaks in the first 1-2 ms after stimulation are stimulus artifacts.

tioning protocol, the final $\mathrm{SOL}_{\mathrm{R}} \mathrm{H}$-reflex is markedly and significantly increased in HRup rats $(p=0.02$, paired $t$ test) and decreased in HRdown rats $(p<0.001)$. In contrast, $\mathrm{SOL}_{\mathrm{R}}$ HRup or HRdown conditioning oppositely affected the $\mathrm{QD}_{\mathrm{R}} \mathrm{H}$-reflex: the final $\mathrm{QD}_{\mathrm{R}} \mathrm{H}$-reflex appears to be smaller in $\mathrm{SOL}_{\mathrm{R}}$ HRup rats $(p=0.06)$, and is larger in $\mathrm{SOL}_{\mathrm{R}} \mathrm{HRdown}$ rats $(p=0.02)$. At the same time, neither $\mathrm{SOL}_{\mathrm{R}}$ HRup or HRdown conditioning has a noticeable effect on $\mathrm{SOL}_{\mathrm{L}} \mathrm{H}$-reflexes. In all rats, $\mathrm{M}$ responses and background EMG do not change.

Figure 2 shows initial and final $\mathrm{SOL}_{\mathrm{R}}$ and $\mathrm{QD}_{\mathrm{R}} \mathrm{H}$-reflexes for an HRup rat (left) and an HRdown rat (right). In the HRup rat, the $\mathrm{SOL}_{\mathrm{R}} \mathrm{H}$-reflex is markedly larger after conditioning, while the $\mathrm{QD}_{\mathrm{R}} \mathrm{H}$-reflex is smaller. Conversely, in the HRdown rat, the $\mathrm{SOL}_{\mathrm{R}} \mathrm{H}$-reflex is much smaller after conditioning, while the $\mathrm{QD}_{\mathrm{R}}$ H-reflex is larger.

\section{Effects of $\mathrm{SOL}_{\mathrm{R}} \mathrm{H}$-reflex conditioning on $\mathrm{SOL}_{\mathrm{R}}$ and $\mathrm{QD}_{\mathrm{R}}$ locomotor H-reflexes}

In 7 of the 9 successful HRup rats and 6 of the 8 successful HRdown rats, $\mathrm{SOL}_{\mathrm{R}}$ and $\mathrm{QD}_{\mathrm{R}}$ locomotor $\mathrm{H}$-reflexes were also studied during the stance phase of locomotion before and after $\mathrm{SOL}_{\mathrm{R}} \mathrm{H}$-reflex conditioning. For these rats, Figure $3 \mathrm{~A}$ shows average final values $( \pm \mathrm{SEM})$ of $\mathrm{SOL}_{\mathrm{R}}$ and $\mathrm{QD}_{\mathrm{R}}$ locomotor $\mathrm{H}$-reflexes in percentage of their initial control values. $\mathrm{SOL}_{\mathrm{R}}$ $\mathrm{H}$-reflex conditioning has effects on the $\mathrm{SOL}_{\mathrm{R}}$ and $\mathrm{QD}_{\mathrm{R}}$ locomotor H-reflexes comparable to its effects on the conditioning $\mathrm{H}$-reflexes. Indeed, the effects on the locomotor H-reflexes are even more prominent. Final $\mathrm{SOL}_{\mathrm{R}}$ locomotor $\mathrm{H}$-reflexes are markedly and significantly increased in HRup rats $(p=0.004)$ and decreased in HRdown rats $(p=0.01)$. In contrast, $\mathrm{SOL}_{\mathrm{R}}$ HRup or HRdown conditioning has an opposite effect on the $\mathrm{QD}_{\mathrm{R}}$ locomotor H-reflex: it appears to be smaller in $\mathrm{SOL}_{\mathrm{R}} \mathrm{HRup}$ rats $(p=0.07)$ and is significantly larger in $\mathrm{SOL}_{\mathrm{R}}$ HRdown rats $(p=0.004)$.

Figure $3 B$ shows initial and final $\mathrm{SOL}_{\mathrm{R}}$ and $\mathrm{QD}_{\mathrm{R}}$ locomotor $\mathrm{H}$-reflexes for one HRup rat (left) and one HRdown rat (right). In the HRup rat, the $\mathrm{SOL}_{\mathrm{R}} \mathrm{H}$-reflex is markedly larger after conditioning, while the $\mathrm{QD}_{\mathrm{R}} \mathrm{H}$-reflex is smaller. Conversely, in the HRdown rat, the $\mathrm{SOL}_{\mathrm{R}} \mathrm{H}$-reflex is smaller after conditioning, while the $\mathrm{QD}_{\mathrm{R}} \mathrm{H}$-reflex is larger. 

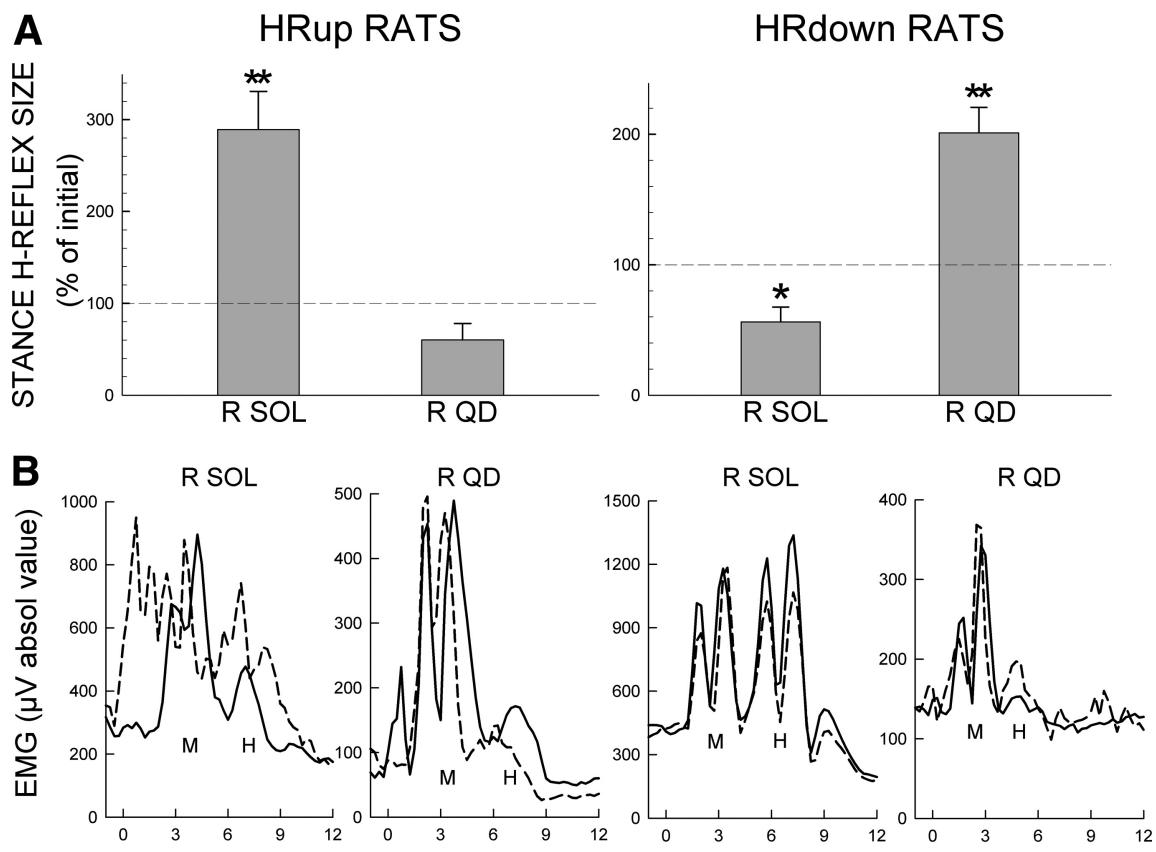

TIME AFTER NERVE STIMULATION (ms)

Figure 3. Effects of $S O \mathrm{~L}_{R}$ HRup and HRdown conditioning on the locomotor H-reflexes. $A$, Average final locomotor H-reflexes (in percentage of their initial values) of $S O L_{R}$ and $Q D_{R}$ muscles for successful HRup (left) and HRdown (right) rats. $p$ values for difference from initial value by paired $t$ test are shown. ${ }^{*} p<0.05$; ${ }^{* *} p<0.01$. B, Effects of $S O L_{R}$ HRup and HRdown conditioning on the locomotor $H$-reflexes in representative rats. Average poststimulus $S O L_{R}$ and $Q D_{R} E M G$ in the stance phase of locomotion during the control-mode (solid) and near the end of conditioning (dashed) for an HRup rat and an HRdown rat. After HRup conditioning, the $S O \mathrm{~L}_{R} \mathrm{H}$-reflex is larger and the $Q D_{R}$ H-reflex is smaller, while after HRdown conditioning, the $\mathrm{SO}_{R} \mathrm{H}$-reflex is smaller and the $\mathrm{QD}_{R} \mathrm{H}$-reflex is larger. Prestimulus EMG (EMG at time 0 ) and M-responses are stable. Peaks in the first $1-2$ ms after stimulation are stimulus artifacts.

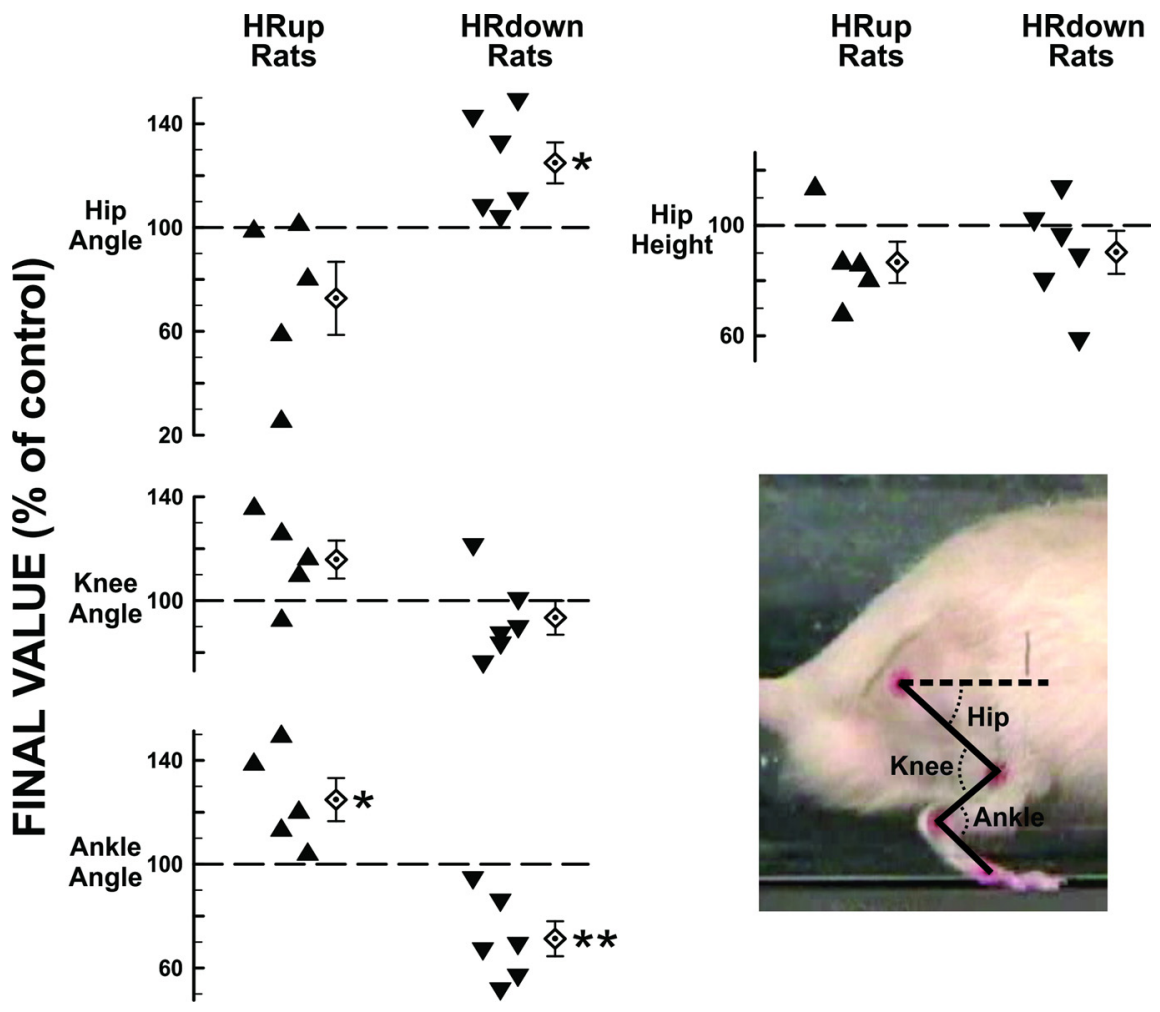

Figure 4. Kinematic effects of $S O L_{R} H R$ up and HRdown conditioning. Individual and average ( $\left.\pm S E M\right)$ final right stance-phase anterior ankle, posterior knee, and anterior hip angles (see inset), and hip height (in percentage of their initial values) for successful HRup (left) and HRdown (right) rats. The dashed horizontal line is parallel to the treadmill surface. $p$ values for difference from the initial value by paired $t$ test are shown $\left({ }^{*} p<0.05 ;{ }^{* *} p<0.01\right)$.
In the unsuccessful rats, final $\mathrm{SOL}_{\mathrm{R}}$ and $\mathrm{QD}_{\mathrm{R}}$ locomotor $\mathrm{H}$-reflexes varied widely across animals. The $\mathrm{SOL}_{\mathrm{R}}$ locomotor $\mathrm{H}$-reflex actually increased in 3 of the 5 unsuccessful HRdown rats, despite the fact that the $\mathrm{SOL}_{\mathrm{R}}$ conditioning H-reflex did not change in any of them. This result is consistent with an earlier study (Chen et al., 2005), and suggests that the lack of change in the conditioning H-reflex does not necessarily mean that conditioning has had no impact (for discussion, see Chen et al., 2005).

Effects of $\mathrm{SOL}_{\mathrm{R}} \mathrm{H}$-reflex conditioning on ankle, knee, and hip angles and hip height

In 5 successful HRup rats and 6 successful HRdown rats, average right ankle, knee, and hip angles and hip height during the stance phase of treadmill locomotion were determined before and after $\mathrm{SOL}_{\mathrm{R}}$ $\mathrm{H}$-reflex conditioning. Figure 4 shows, for each rat, the final values ( \pm SEM) of these measures in percentage of their initial values. In HRup rats, final anterior ankle angles were larger $(p=0.04)$ (i.e., the ankle was more plantarflexed) and final anterior hip angles tended to be smaller (i.e., the hip was less extended) $(p=0.13)$. In contrast, in HRdown rats, final ankle angles were smaller $(p=0.008)$ and final hip angles were larger $(p=0.03)$. Neither HRup nor HRdown conditioning significantly affected knee angle or hip height.

\section{Discussion}

Operant conditioning of the rat $\mathrm{SOL}_{\mathrm{R}}$ H-reflex has an opposite effect on the $\mathrm{QD}_{\mathrm{R}}$ H-reflex: in $\mathrm{SOL}_{\mathrm{R}}$ HRup rats the $\mathrm{QD}_{\mathrm{R}} \mathrm{H}$-reflex usually goes down, and in $\mathrm{SOL}_{\mathrm{R}}$ HRdown rats the $\mathrm{QD}_{\mathrm{R}} \mathrm{H}$-reflex goes up. These $\mathrm{QD}_{\mathrm{R}} \mathrm{H}$-reflex changes, like the $\mathrm{SOL}_{\mathrm{R}}$ changes, are still present, perhaps even greater, during locomotion. They occur despite the fact that they do not affect reward probability; the $\mathrm{QD}_{\mathrm{R}}$ $\mathrm{H}$-reflex is elicited at different times from the $\mathrm{SOL}_{\mathrm{R}} \mathrm{H}$-reflex and is never associated with a reward. Nevertheless, it changes. Furthermore, $\mathrm{SOL}_{\mathrm{R}} \mathrm{H}$-reflex conditioning has effects on ankle angle during locomotion that are consistent with the $\mathrm{SOL}_{\mathrm{R}}$ $\mathrm{H}$-reflex changes; and, in addition, it has opposite effects on hip angle, the etiology of which is not known at present.

The plasticity responsible for change in the $\mathrm{QD}_{\mathrm{R}} \mathrm{H}$-reflex

The $\mathrm{QD}_{\mathrm{R}} \mathrm{H}$-reflex is the earliest possible CNS-mediated QD response to the $\mathrm{PF}_{R}$ nerve stimulus. Thus, the changes in $\mathrm{QD}_{\mathrm{R}}$ $\mathrm{H}$-reflex size associated with $\mathrm{SOL}_{\mathrm{R}} \mathrm{H}$-reflex 
conditioning could reflect plasticity in the reflex pathway itself or plasticity in neurons or synapses that provide tonic input to the pathway, input that is there before the nerve stimulus. At present, evaluation of these two possibilities rests mainly on what is known about the change in the H-reflex that controls the reward (i.e., the conditioned H-reflex).

Physiological and anatomical studies [for review, see Wolpaw and Chen (2009) and Wolpaw (2010)] indicate that the change in the reflex being conditioned (i.e., the $\mathrm{SOL}_{\mathrm{R}} \mathrm{H}$-reflex) is due mainly to plasticity in the motoneuron and/or the afferent pathway from the nerve stimulus, and that this plasticity is caused by change in corticospinal tract (CST) activity (which may pass through GABAergic interneurons in spinal laminae 6 and 7). For example, down-conditioning appears to be due mainly to a positive shift in motoneuron firing threshold (Carp and Wolpaw, 1994). Furthermore, the change in the conditioned reflex is still evident when tonic inputs are greatly reduced or entirely eliminated (Wolpaw and Lee, 1989).These findings suggest that the $\mathrm{QD}_{\mathrm{R}} \mathrm{H}$-reflex change reflects comparable plasticity in the $\mathrm{QD}_{\mathrm{R}}$ $\mathrm{H}$-reflex pathway. This conclusion is supported by the fact that the $\mathrm{QD}_{\mathrm{R}} \mathrm{H}$-reflex change is still evident during locomotion, which would be expected to modify tonic inputs.

\section{The etiology of the change in the $\mathrm{QD}_{\mathrm{R}} \mathrm{H}$-reflex and in hip angle}

The plasticity that changes the $\mathrm{QD}_{\mathrm{R}} \mathrm{H}$-reflex or hip angle might occur in two ways. First, it might be an inevitable consequence of the fact that the capacity for activity-dependent plasticity is ubiquitous in the CNS. For example, the same CST activity that changes the $\mathrm{SOL}_{\mathrm{R}} \mathrm{H}$-reflex pathway might also produce an opposite change in the $\mathrm{QD}_{\mathrm{R}} \mathrm{H}$-reflex pathway. Such inverse effects on different muscles can occur with supraspinal lesions (e.g., Thompson et al., 2009b). Furthermore, the plasticity in the $\mathrm{SOL}_{\mathrm{R}}$ $\mathrm{H}$-reflex pathway, by changing ongoing activity in intraspinal pathways, might itself induce plasticity in the $\mathrm{QD}_{\mathrm{R}} \mathrm{H}$-reflex. Comparable plasticity in spinal pathways that affect hip muscles might account for the change in hip angle. Plasticity created in such ways reflects the activity-dependent properties of individual neurons and synapses. Thus, its etiology is local, and it can be called "reactive" plasticity (Wolpaw, 1997). A simple example of reactive plasticity is synaptic desensitization caused by increased synaptic input (e.g., Otis et al., 1996).

The second possible etiology of the $\mathrm{QD}_{\mathrm{R}} \mathrm{H}$-reflex change or the hip angle change is that it is adaptive, that it helps to compensate for the impact of $\mathrm{SOL}_{\mathrm{R}} \mathrm{H}$-reflex conditioning on other behaviors. Because the spinal cord is the final common pathway for many behaviors, the plasticity in the $\mathrm{SOL}_{\mathrm{R}} \mathrm{H}$-reflex pathway that increases reward probability affects other behaviors that also use this pathway. Indeed, $\mathrm{SOL}_{\mathrm{R}} \mathrm{H}$-reflex conditioning can be used to improve locomotion after a partial spinal cord injury (Y. Chen et al., 2006). In normal rats with normal locomotion, such side effects of H-reflex conditioning may induce additional activity-dependent plasticity that preserves normal locomotion (or other important behaviors). Chen et al. (2005) found that conditioning of the $\mathrm{SOL}_{\mathrm{R}}$ $\mathrm{H}$-reflex changed SOL locomotor activity, but did not affect stepcycle length or symmetry, suggesting that other changes had preserved these major parameters of the step-cycle. This additional plasticity can be called "compensatory" (Wolpaw, 1997). Unlike reactive plasticity, which originates locally, compensatory plasticity is induced and shaped by interactions between the CNS and the external world (Wolpaw, 2010 for discussion).

The present kinematic results suggest that $\mathrm{SOL}_{\mathrm{R}} \mathrm{H}$-reflex conditioning does produce compensatory plasticity. $\mathrm{SOL}_{\mathrm{R}} \mathrm{H}$-reflex conditioning had effects on stance-phase ankle plantarflexion that are consistent with the $\mathrm{SOL}_{\mathrm{R}} \mathrm{H}$-reflex change: increase in HRup rats and decrease in HRdown rats. At the same time, it had opposite effects on hip extension: decrease in HRup rats and increase in HRdown rats. These opposite changes in hip angle appear to explain why hip height was not significantly changed despite the changes in ankle angle (i.e., Fig. 4).

A unilateral change in hip height during locomotion would presumably twist the thorax, which would probably have widespread short-term and long-term musculoskeletal effects. Nociceptive or other sensory inputs produced by this twisting might operantly condition compensatory plasticity that eliminates the twisting and preserves hip height. Thus, like step-cycle symmetry (Chen et al., 2005), hip-height symmetry during locomotion may be a functionally important parameter; and an intervention that disrupts it, such as $\mathrm{SOL}_{\mathrm{R}} \mathrm{H}$-reflex conditioning (which changes stance-phase foot plantarflexion), may induce compensatory plasticity that prevents the disruption. Whether the change in hip angle does reflect compensatory plasticity, whether reflex changes account for it, and why knee angle does not change instead (or in addition) are questions that will hopefully be elucidated by the comprehensive kinematic and reflex studies now underway (Liu et al., 2010).

\section{Therapeutic applications of spinal reflex conditioning}

H-reflex conditioning can improve locomotion in rats after a partial spinal cord injury (Y. Chen et al., 2006). Initial studies suggest that it can be effective in humans with spinal cord injuries, and they indicate that it requires only a small fraction of the conditioning trials normally used in animals (Thompson et al., 2009a; Pomerantz et al., 2010). The ability to target specific pathways could enable reflex conditioning protocols to supplement other therapeutic interventions such as locomotor training (Harkema et al., 2011). These protocols could be particularly useful when spinal cord regeneration becomes possible and methods are needed for guiding plasticity to produce a functionally effective spinal cord.

In the context of such therapeutic possibilities, the present results are both sobering and encouraging. They indicate the complexity of the effects that might accompany this new approach. At the same time, by suggesting that the plasticity induced by reflex conditioning may target the preservation (or restoration) of important functional parameters (e.g., hip height), they encourage further exploration of its therapeutic applications.

\section{Conclusions}

Soleus H-reflex conditioning also affects the H-reflex of the quadriceps muscle group, which operates about different joints, and it changes locomotor kinematics at both the ankle and the hip. The quadriceps H-reflex change remains evident during locomotion, and is probably due to plasticity in that H-reflex pathway. The change in hip angle is likely to reflect compensatory plasticity that preserves hip height despite the change in ankle angle. These results are striking evidence of the complex effects of acquiring an ostensibly simple skill. Their further study may illuminate the etiology and functional impact of the complex plasticity associated with new skills, and may guide development of new methods to improve function after trauma or disease.

\section{References}

Carp JS, Wolpaw JR (1994) Motoneuron plasticity underlying operantly conditioned decrease in primate H-reflex. J Neurophysiol 72:431-442. 
Chen XY, Wolpaw JR (1995) Operant conditioning of H-reflex in freely moving rats. J Neurophysiol 73:411-415.

Chen XY, Wolpaw JR (2002) Probable corticospinal tract control of spinal cord plasticity in rats. J Neurophysiol 87:645-652.

Chen XY, Chen L, Chen Y, Wolpaw JR (2006) Operant conditioning of reciprocal inhibition in rat soleus muscle. J Neurophysiol 96:2144-2150.

Chen Y, Chen XY, Jakeman LB, Schalk G, Stokes BT, Wolpaw JR (2005) The interaction of a new motor skill and an old one: $\mathrm{H}$-reflex conditioning and locomotion in rats. J Neurosci 25:6898-6906.

Chen Y, Chen XY, Jakeman LB, Chen L, Stokes BT, Wolpaw JR (2006) Operant conditioning of $\mathrm{H}$-reflex improves locomotion after spinal cord injury in rats. J Neurosci 26:12537-12543.

Harkema S, Behrman A, Barbeau H (2011) Locomotor training. Principles and practice. New York: Oxford UP.

Liu RL, Chen Y, Chen L, Wang Y, Wolpaw JR, Chen XY (2010) Effects of soleus H-reflex conditioning on hip, knee, and ankle extensor and flexor muscles: methods and initial results. Soc Neurosci Abstr 36:82.18.

Otis T, Zhang S, Trussell LO (1996) Direct measurement of AMPA receptor desensitization induced by glutamatergic synaptic transmission. J Neurosci 16:7496-7504.

Pomerantz F, Wolpaw JR, Lichtman SW, Abel B, DeFrancesco E, Thompson AK (2010) Operant down conditioning of the soleus $\mathrm{H}$-reflex in people with spasticity due to chronic incomplete spinal cord injury. Soc Neurosci Abstr 36:82.14.

Thompson AK, Chen XY, Wolpaw JR (2009a) Acquisition of a simple skill: task-dependent adaptation plus long-term change in the human soleus H-reflex. J Neurosci 29:5784-5792.

Thompson AK, Estabrooks KL, Chong S, Stein RB (2009b) Spinal reflexes in ankle flexor and extensor muscles after chronic central nervous system lesions and functional electrical stimulation. Neurorehabil Neural Repair 23:133-142.

Wolpaw JR (1987) Operant conditioning of primate spinal reflexes: the $\mathrm{H}$ reflex. J Neurophysiol 57:443-459.

Wolpaw JR (1997) The complex structure of a simple memory. Trends Neurosci 20:588-594.

Wolpaw JR (2010) What can the spinal cord teach us about learning and memory? Neuroscientist 16:532-549.

Wolpaw JR, Chen XY (2009) Operant conditioning of reflexes. In: Encyclopedia of neuroscience (Squire LR, ed), Vol 7, pp 225-233. Oxford: Academic.

Wolpaw JR, Lee CL (1989) Memory traces in primate spinal cord produced by operant conditioning of H-reflex. J Neurophysiol 61:563-572.

Wolpaw JR, Braitman DJ, Seegal RF (1983) Adaptive plasticity in the primate spinal stretch reflex: initial development. J Neurophysiol 50:12961311. 